\title{
Generation of Electron and Fast Atom Beams by a Grid Immersed in Plasma
}

\author{
Alexander Metel ${ }^{1, *}$, Enver Mustafaev ${ }^{1}$, Yury Melnik ${ }^{1}$, and Khaled Hamdy ${ }^{2}$ \\ ${ }^{1}$ Moscow State Technological University "STANKIN", RU-127055, Moscow, Russia \\ ${ }^{2}$ Production Engineering and Mechanical Design Department, Faculty of Engineering, Minia University, EG-61519, Minia, Egypt
}

\begin{abstract}
We present a new method of product processing with beams of accelerated electrons and fast neutral atoms, which are generated by an immersed in plasma grid under a high negative voltage of $5 \mathrm{kV}$. The electrons appear due to secondary emission from the grid surface provoked by its bombardment with ions accelerated from the plasma. At the gas pressure not exceeding $0.1 \mathrm{~Pa}$ the ions with energy of $5 \mathrm{keV}$ reach the grid without collisions in the space charge sheaths near its surface and their current in the grid circuit is by 2-3 times lower than the electron current. At higher pressures accelerated ions due to charge exchange collisions in the sheaths turn into fast neutral atoms leaving the sheaths and forming the beams. With the pressure increasing, the electron beam current diminishes and the current of fast atom beam grows.
\end{abstract}

\section{Introduction}

The ion-plasma processing of engineering products allows a modification of their surface properties. Using the plasma immersion ion implantation [1-3], materials can be modified and alloying additives can be introduced into the surface layer. Using sources of metal plasma [4] or metal vapor [5], it allows synthesis of wear-resistant coatings on the products. Ion-plasma treatment reduces the coefficient of friction, increases the useful life of the products, significantly increases their wear resistance and corrosion resistance. It also allows for combined treatment, for example, ion nitriding of products $[6,7]$ with subsequent deposition of wear-resistant coatings.

In the latter case, a hardened nitride surface layer with a thickness of 50-100 $\mu \mathrm{m}$ prevents brittle fracture of hard coatings with a thickness not exceeding $5 \mu \mathrm{m}$. This improves the adhesion of the coating and its resistance to cracking. Hence, the product combines wear resistance with high load bearing capacity and good fatigue strength. In the plasma-immersion ion implantation, the product is immersed in a plasma containing necessary ions, and negative high-voltage pulses are applied to it [8-10]. The product surface bombardment by ions accelerated from the plasma appreciably improves the coating properties [11, 12].

It is impossible to apply high-voltage pulses to dielectric products and for this reason, broad beams of accelerated ions $[13,14]$ or fast neutral atoms [15, 16] are used to bombard the product surface. The beam sources are also applied to bombard the coating during its deposition and for etching the product surface [17]. All kinds of processing are carried out in discharge plasma at a gas pressure of 0.05-1 $\mathrm{Pa}$ [18].
It is possible to dispense with beam sources using an immersed in plasma grids under negative bias potential. Accelerated from the plasma ions will pass through the grid holes and return back on the other side of the grid. At an appropriate gas pressure, they can turn into fast neutral atoms due to charge exchange collisions in the space charge sheaths of the grid. Only fast atoms can leave the sheaths and form neutral beams. It is obvious that the presumable method for generation of fast atom beams should be cheaper in realisation as compared to application of beam sources.

In any case, it is necessary first to develop a model of the beam generation allowing for prediction of the beam parameters dependence on the gas pressure and the discharge current.

\section{Beams produced by a flat grid}

Let us consider a flat titanium grid 1 (Figure 1) installed in a vacuum chamber filled with plasma 2 and 3 of the glow discharge with electrostatic electron confinement [18] at an argon pressure of $0.1 \mathrm{~Pa}$. When a negative voltage $U$ is applied to the grid, sheaths 4 and 5 of the positive space charge of ions 6 and 7 accelerated from the plasma are formed between the grid and the plasma the sheath width amounting to $d$.

The current density $j_{i}$ of these ions on the surface of grid 1 is determined by the emission capacity of the plasma and is expressed by the Bohm formula [19]:

$$
\begin{aligned}
j_{\mathrm{i}}=(2 \mathrm{e})^{-1 / 2} \text { en }\left(2 k T_{\mathrm{e}} / M\right)^{1 / 2}= & \\
& =0.94 \times 10^{-15} n\left[T_{\mathrm{e}}(\mathrm{eV}) / M_{\mathrm{A}}\right]^{1 / 2},
\end{aligned}
$$

where e is the Naperian base, $n$ is the plasma density, $T_{\mathrm{e}}$ is the temperature of plasma electrons, $e$ is the

Corresponding author: a.metel@stankin.ru 
elementary charge, $k$ is the Boltzmann constant, $M$ is the ion mass in kilograms, and $M_{\mathrm{A}}$ is the ion mass in atomic mass units $A=1.66 \times 10^{-27} \mathrm{~kg}$. For a plasma with a density of $n=2 \times 10^{16} \mathrm{~m}^{-3}$ and an electron temperature $T_{\mathrm{e}}$ $=1 \mathrm{eV}$, the ion current density on the grid is equal to $j_{\mathrm{i}}=$ $3.2 \mathrm{~A} / \mathrm{m}^{2}$.

Fig. 1. Schematic of broad electron and fast atom beams

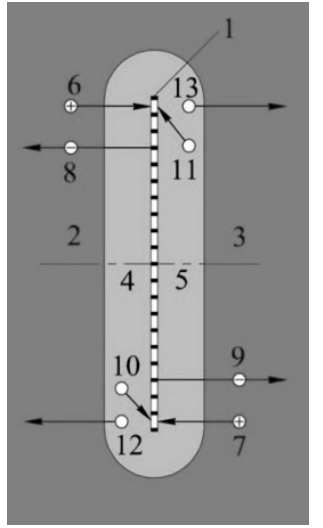

generation using a flat grid immersed in plasma under negative voltage.

If we take into account the coefficient of secondary electron emission $\gamma_{i}=1.3$ for argon ions with an energy of $4 \mathrm{keV}$, then the current density of electrons 8 and 9 emitted by the grid is equal to $j_{\mathrm{e}}=\gamma_{\mathrm{i}} j_{\mathrm{i}}=1.3 \times 3.2 \mathrm{~A} / \mathrm{m}^{2} \approx$ $4.2 \mathrm{~A} / \mathrm{m}^{2}$, and the total current density of ions and electrons on the surface of the grid amounts to $j=$ $j_{\mathrm{i}}\left(1+\gamma_{\mathrm{i}}\right)=7.4 \mathrm{~A} / \mathrm{m}^{2}$. The width $d$ of the space charge sheaths is determined by the Child-Langmuir expression [20]:

$$
j_{\mathrm{i}}=(4 / 9) \varepsilon_{\mathrm{o}}(2 e / M)^{1 / 2} U^{3 / 2} / d^{2},
$$

If the ion mass $M=A M_{\mathrm{A}}$ is expressed in atomic mass units $A=2.7 \cdot 10^{-11} \mathrm{~kg}$, then the ion current density

$$
\left.j_{\mathrm{i}}=5.46 \times 10^{-8} 1 / M_{\mathrm{A}}\right)^{1 / 2} \mathrm{U}^{3 / 2} / d^{2}
$$

With a grid voltage $U=4000 \mathrm{~V}, M_{\mathrm{A}}=40$ and $j_{\mathrm{i}}=$ $3.2 \mathrm{~A} / \mathrm{m}^{2}$ we get $d^{2}=6.82 \times 10^{-4} \mathrm{~m}^{2}$ and $d=2.6 \times 10^{-2} \mathrm{~m}$. At a pressure of $0.1 \mathrm{~Pa}$, the sheath width $d=2.6 \mathrm{~cm}$ is less than the mean free path of gas molecules $\lambda \approx 7 \mathrm{~cm}$ and less than the ion charge exchange length $\lambda_{\mathrm{c}} \sim 20 \mathrm{~cm}$. Therefore, accelerated from plasma 2 and 3 ions 6 and 7 fly to the grid surface without collisions.

Let us consider the reciprocation of ions in the sheaths. To determine the average path of $N$ ions in the space charge sheaths prior to absorption by the grid, we divide them into groups of ions with equal path lengths $l_{\mathrm{k}}(\mathrm{k}=1,2,3 \ldots)$. At the transparency $\eta$ of the grid $N(1-\eta)$ of ions are absorbed by the grid, and $N \eta$ of ions fly through its holes, slowdown and turn back to fly in the opposite direction. Hence, the path of the first group of ions in the amount of $N(1-\eta)$ is equal to $l_{1}=d$.

When $N \eta$ of ions move backward through the grid, $N \eta(1-\eta)$ of ions are absorbed, and $N \eta^{2}$ of ions fly through its holes, slowdown and turn back for a flight in the opposite direction. Therefore, the path length of the second group of ions in the amount of $N \eta(1-\eta)$ is equal to $l_{2}=3 d$.

When $N \eta^{\mathrm{k}-1}$ of ions move backward through the grid, $N \eta^{\mathrm{k}-1}(1-\eta)$ of them are absorbed by the grid, while $\mathrm{N} \eta^{\mathrm{k}}$ of ions fly through its holes, slowdown and turn around to fly in the opposite direction. Therefore, the path length of the k-th group in the amount of $N \eta^{\mathrm{k}-1}(1-\eta)$ is equal to $l_{\mathrm{k}}=(2 \mathrm{k}-1) d$.

To obtain the average path length of ions in the sheaths, it is necessary to average the path lengths $l_{\mathrm{k}}$ of all groups according to the number of ions in the groups. Therefore, the average path length of ions reciprocating in the sheaths is equal to

$$
\begin{aligned}
L= & {\left[l_{1} N(1-\eta)+l_{2} N \eta(1-\eta)+\ldots+l_{\mathrm{k}} N \eta^{\mathrm{k}-1}(1-\eta)+\ldots\right] / N=} \\
= & (1-\eta)\left[d+3 d \eta+5 d \eta^{2}+\ldots+(2 \mathrm{k}-1) d \eta^{\mathrm{k}-1}+\ldots\right]= \\
& =d(1-\eta)\left[2 \sum_{\mathrm{k}=1}^{\infty} \mathrm{k} \eta^{\mathrm{k}-1}-\sum_{\mathrm{k}=1}^{\infty} \eta^{\mathrm{k}-1}\right] .
\end{aligned}
$$

Taking into account that $\sum_{\mathrm{k}=1}^{\infty} \eta^{\mathrm{k}-1}=(1-\eta)^{-1}$, and $\sum_{\mathrm{k}=1}^{\infty} \mathrm{k} \eta^{\mathrm{k}-1}=(1-\eta)^{-2}$, the average path of ions in the space charge sheaths amounts to

$$
L=d(1+\eta)(1-\eta)^{-1}
$$

In our case, at $d=2.6 \mathrm{~cm}$ and $\eta=0.8$, the average path of accelerated ions in the sheaths amounts to $L=$ $23.4 \mathrm{~cm}$. At argon pressure of $0.01 \mathrm{~Pa}$ the ion charge exchange length $\lambda_{\mathrm{c}} \sim 2 \mathrm{~m}$ by an order of magnitude exceeds $L$. Therefore, no charge exchange collisions occur in the sheaths, and no fast atoms are produced. Only two beams of secondary electrons with energy $e U$ $=4 \mathrm{keV}$ and current density $j_{\mathrm{e}}=4.2 \mathrm{~A} / \mathrm{m}^{2}$ are formed, which propagate in opposite directions. When the pressure grows to $0.1 \mathrm{~Pa}, \lambda_{\mathrm{c}}$ diminishes to $\sim 20 \mathrm{~cm}$ and at $\lambda_{\mathrm{c}}<L$ fast atoms appear.

The energy e $\varphi$ of a fast neutral atom corresponds to the potential $\varphi$ of the point of its production in the sheath with a width of $d$ at a distance $x$ from the grid surface. The potential distribution in the sheath is described by the expression [19]:

$$
\varphi=U(1-x / d)^{4 / 3}
$$

The maximum energy $\sim e U$ is possessed by fast atoms formed directly in the grid holes, i.e. at $\mathrm{x} \approx 0$ and $\varphi$ equal to the potential difference $U$ between the plasma and the grid (Figure 1). With increasing distance $x$ from the grid, the energy of atoms decreases to zero at $x=d$ at the boundary of the discharge plasma.

With a further increase in pressure, the ions accelerated from the plasma turn into fast atoms even before crossing the plane of the grid. On average, at a distance from the plasma boundary equal to the charge exchange length $\lambda_{c}$, the accelerated ion becomes a fast atom and a slow ion appears. The latter is accelerated in the electric field, travels the same distance $\sim \lambda_{c}$ and turns into the second fast atom. As a result, one ion with the energy $e U \lambda_{c} / d$ and $\left(d / \lambda_{c}-1\right)$ fast atoms with the same energy come to the grid. For this reason, the power density of ions on the grid and proportional to the power density current density $j_{\mathrm{e}}$ of electron beams decrease 
with increasing pressure, while the power transported by fast atoms increases. This means that with increasing pressure, the equivalent current of fast argon atoms increases, while the current of electron beams and the intensity of the grid sputtering decrease.

When replacing a flat grid with a concave one (Figure 2), beams are formed in the same way. However, the trajectories of ions 2, accelerated from plasma 3 in sheath 4 at the convex surface of grid 1, as well as fast atoms 6 produced in collisions with gas molecules 5 converge at its focal point.

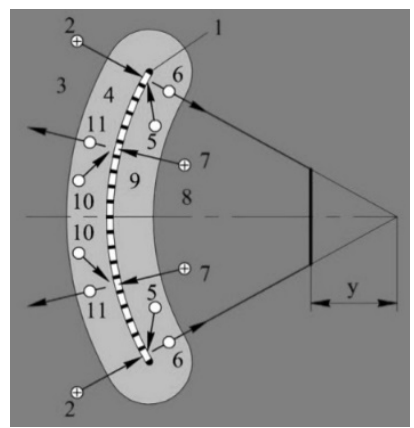

Fig. 2. Generation of compressed beam of fast atoms using a grid with concave surface.

With an increase in the distance from the center of the concave surface of the grid 1, the flow density of fast atoms increases. This makes it possible to multiply the rate of the substrate sputtering in comparison with a flat grid and to increase the heating temperature of the substrate.

\section{Experimental study of the fast atom beam formation by a concave grid}

The verification of the theoretically obtained regularities of beam formation was carried out using a system shown in Figure 3. A round 20-cm-diameter grid with a 20-cmcurvature radius of its concave surface was fixed to a high-voltage feedthrough inside the system chamber. On the grid surface, holes with a diameter of $7 \mathrm{~mm}$ are evenly distributed at a distance of $8 \mathrm{~mm}$ between their centers. In the focus of the grid at a distance of $6 \mathrm{~cm}$ from the chamber wall, a holder for the samples to be processed by the beam is installed.

At an argon pressure in the chamber of $p \sim 0.5 \mathrm{~Pa}$, an increase in the voltage between the anode and the chamber to several hundred volts leads to breakdown and the establishing of a glow discharge with a current in the anode circuit $I_{\mathrm{a}}$ up to 4 A and a voltage of $U_{\mathrm{d}}=400$ $500 \mathrm{~V}$. The chamber turns filled with a brightly glowing homogeneous plasma, separated from its walls by a cathode sheath, and from the grid - by a grid sheath of theion space charge. When the camera and the grid are equipotential, both space charge sheaths are of the same width.

The voltage between the chamber and the anode is independent of the argon pressure $p$ at a constant current $I_{\mathrm{a}}$ in the anode circuit and the pressure ranging from 0.2 to $1 \mathrm{~Pa}$. However, at $p<0.2 \mathrm{~Pa}$, a further decrease in pressure causes an increase in the discharge voltage $U_{\mathrm{d}}$,

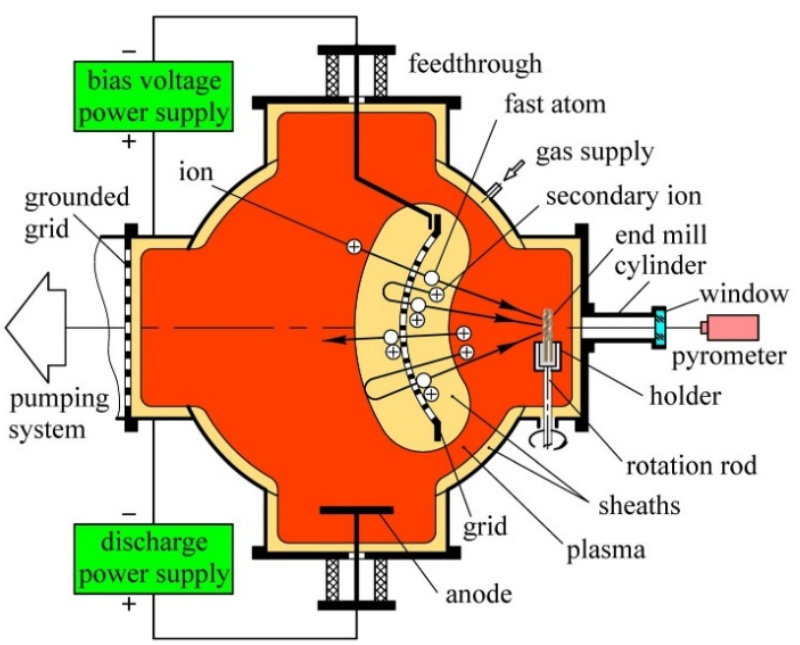

and at $\mathrm{p}=0.02 \mathrm{~Pa}$ it reaches a value of $U_{\mathrm{d}} \sim 1 \mathrm{kV}$.

Fig. 3. Schematic of the beam formation by a grid.

With an increase in the voltage $U$ between the chamber and the grid from zero to $5 \mathrm{kV}$, the current $I$ in the grid circuit at a constant current $I_{\mathrm{a}}$ in the anode circuit approximately doubles, the width $d$ of the sheath between the plasma and the grid increases to $5-10 \mathrm{~cm}$, and the discharge voltage $U_{\mathrm{d}}$ decreases approximately two times.

The density of gas atoms at room temperature and pressure $p=0.02 \mathrm{~Pa}$ is equal to $n=0.5 \times 10^{19} \mathrm{~m}^{-3}$ [21] and the mean path length of argon ions between collisions with charge exchange, called the charge exchange length, is $\lambda=1 / \mathrm{n} \sigma=1 \mathrm{~m}$. Here we took into account that the cross section of charge exchange collisions for argon ions with an energy of $5 \mathrm{keV}$ is equal to $\sigma=2 \times 10^{-19} \mathrm{~m}^{2}$ [22-23].

The width of the grid sheath $\mathrm{d} \sim 0.05 \mathrm{~m}$ is much less than the recharging length $\lambda c$, and therefore the formation of fast atoms in the sheath does not occur. All ions drawn from the plasma bombard the grid and cause secondary electron emission from its surface.

At a pressure of $p=0.02 \mathrm{~Pa}$, the grid emits only electron beams with an energy of $e\left(U+U_{\mathrm{d}}\right)$, propagating from the accelerating grid in opposite directions. Their total current $I_{e}=I \gamma_{\mathrm{i}} /\left(1+\gamma_{\mathrm{i}}\right)$ is approximately equal to $0.63 I$, where $I$ is the current in the grid circuit, and $\gamma_{i}=$ 1.7 is the coefficient of ion-electron emission. The surface of the plasma emitting ions onto the concave surface of the grid is less than the surface of the plasma emitting ions onto its convex surface (Fig. 3). Therefore the current of the electron beam diverging to the chamber walls is higher than the current of the electron beam converging on the sample.

With an increase in pressure to $0.2 \mathrm{~Pa}$, accelerated ions turn in the sheath into fast atoms escaping from the sheath. The energy of each of them, e $\varphi$, corresponds to the potential $\varphi$ of the point in the sheath in which it appeared.

In this regard, the energy of fast atoms varies from zero to $e\left(U+U_{\mathrm{d}}\right)$. The secondary ions formed as a result 
of charge exchange collisions are accelerated in the sheath and also turn into fast neutral atoms that form a beam.

With an increase in pressure $p$ from 0.2 to $2 \mathrm{~Pa}$, the number of fast atoms increases tenfold, their energy decreases from $5 \mathrm{keV}$ to $500 \mathrm{eV}$, and the currents of neutral beams increase.

As for the accelerated electrons, their current decreases with increasing pressure, their energy is equal to $5 \mathrm{keV}$ and practically does not change over the entire range from 0.02 to $2 \mathrm{~Pa}$. Measurement of the voltage between the anode and the holder showed that its potential does not exceed $20 \mathrm{~V}$.

With increasing pressure $p$, the current in the grid circuit increases to $I=0.36$ A at $p=0.4$ and to $I=0.48$ A at $p=1.5 \mathrm{~Pa}$. There is also a decrease in the discharge voltage to $U_{\mathrm{d}}=170 \mathrm{~V}$ at $p=0.4$ and to $U_{\mathrm{d}}=55 \mathrm{~V}$ at $p=$ $1.5 \mathrm{~Pa}$.

When a 1-mm-thick flat titanium target is placed in the grid focus, a 2-cm-diameter brightly glowing circle is observed on its surface heated by the fast atom beam. The temperature grows with increasing the gas pressure $p$ and changes with the distance from the left edge of the target, reaching the maximum value of $700^{\circ} \mathrm{C}$ at $p=0.4$ and $900^{\circ} \mathrm{C}$ at $p=1.5 \mathrm{~Pa}$ in the center of the glowing circle.

An increase in the heating intensity with increasing pressure confirms the theory's conclusion about an increase in the flux of fast neutral atoms with a decrease in the charge exchange length $\lambda$ of ions below the width $d$ of the space charge sheath near the grid.

Fast atoms propagating from the concave surface of the 200-mm-diameter grid bombard the target within a circle with a diameter not exceeding $10 \mathrm{~mm}$. However, due to thermal conductivity, the width of the target temperature distribution must exceed the width of the neutral atoms flux distribution.

For a more accurate estimate of the beam diameter, we measured the distribution of the etching rate by fast atoms of a polished titanium target with a mask on its surface. The straight boundary between them was located horizontally (Fig. 4).

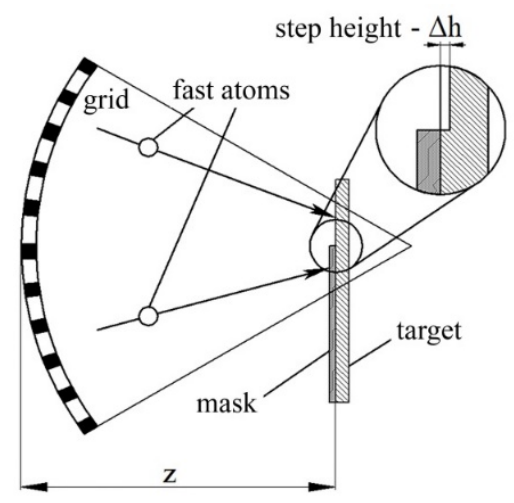

Fig. 4. The formation of a step on the surface of the target installed at a distance $\mathrm{z}$ from the grid, due to bombardment with a beam of fast argon atoms.

The target was bombarded with fast atoms at a current in the anode circuit $I_{\mathrm{a}}=1 \mathrm{~A}$ and an argon pressure $p=1.5 \mathrm{~Pa}$. Then using a mechanical profile meter Dektak XT the height difference between the covered with the mask and the open substrate surface was measured along the border between them.

The sputtering rate of the target surface was determined by dividing the height of the step by the time of its bombardment with fast argon atoms. The measurement results presented in Figure 5 made it possible to determine the effective beam diameter of fast neutral argon atoms.

Fig. 5. The distribution of the target etching rate over

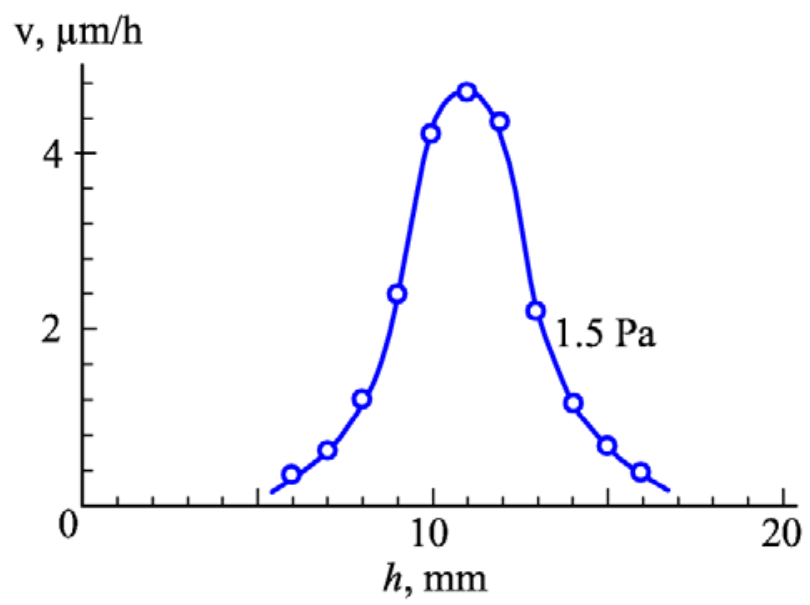

itssurfaces at a the grid voltage of $U=5 \mathrm{kV}$, the argon pressure of $1.0 \mathrm{~Pa}$ and the anode current of $I_{\mathrm{a}}=1 \mathrm{~A}$.

Since the sputtering rate $\mathrm{v}$ is proportional to the flux density of fast atoms, the width of the distribution shown in Figure 5 gives an effective diameter $D$ of the fast atom beam equal to $5 \mathrm{~mm}$ in the focal plane of the grid at a distance of $20 \mathrm{~cm}$ from its center.

Figure 6 shows the dependence of the effective beam diameter $\mathrm{D}$ on the distance $Z$ between the target and the grid. The diameter values were determined in a similar way from the distribution of the sputtering rate of the flat polished substrates.

As the distance $Z$ between the target and the grid increases, the diameter $D$ decreases from $45 \mathrm{~mm}$ at $Z=$ $17 \mathrm{~cm}$ to $6 \mathrm{~mm}$ at $Z=20 \mathrm{~cm}$, and as the distance increases from $Z=21 \mathrm{~cm}$ to $Z=24 \mathrm{~cm}$, the diameter $D$ increases from $7 \mathrm{~mm}$ up to $52 \mathrm{~mm}$.

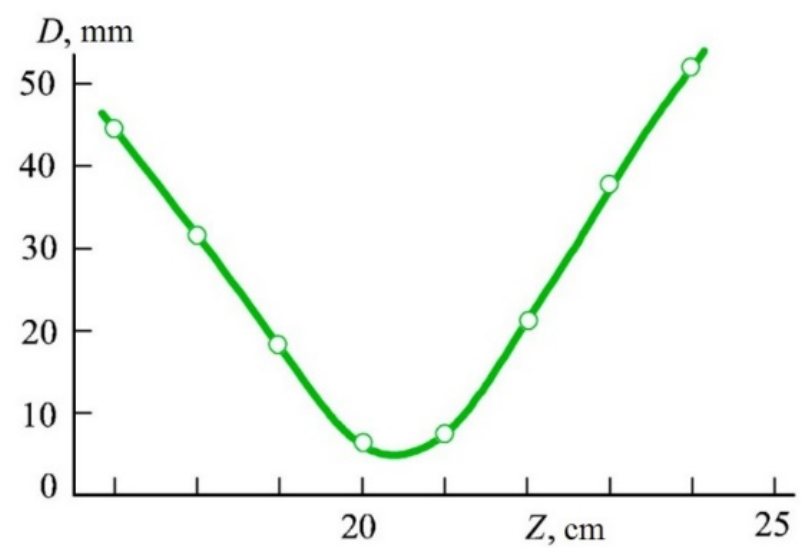


Fig. 6. The dependence of the beam diameter $\mathrm{D}$ on the distance $\mathrm{Z}$ between the grid and the target.

This means that by changing the distance between the grid and a product to be processed, it is possible to achieve that the beam diameter exceeds the overall dimensions of the product rotating in the plasma, and that fast atoms bombard its entire surface.

The experimental results show that the processing with a grid immersed in plasma is universal. At the gas pressure not exceeding $0.1 \mathrm{~Pa}$, it allows heating the products with electron beams without etching their surfaces. At the gas pressure exceeding $1 \mathrm{~Pa}$, it allows a uniform etching of the product surface with fast atoms without blunting its sharp edges. The surface etching rate depends on the product material and fast atoms energy [24]. The new method for surface processing with fast atoms can replace the methods for plasma electrolytic polishing [25], laser-plasma polishing of metals [26], surface polishing with pulsed electron beams [27] and also can be used for micro- and nanoelectronics [28].

\section{Acknowledgments}

This work was supported by the Russian Science Foundation under grant 20-19-00620. This work was carried out using equipment provided by the Center of Collective Use of MSUT "STANKIN".

\section{References}

1. Handbook of Plasma Immersion Ion Implantation and Deposition, A. Anders (Ed.) (JohnWiley \& Sons, Inc., New York, 2000)

2. N.N. Koval, A.I. Ryabchikov, D.O. Sivin, I.V. Lopatin, O.V. Krysina, Yu.H. Akhmadeev, D.Yu. Ignatov, Surf. Coat. Technol., 340, 152 (2018)

3. D. Vempaire, J. Pelletier, A. Lacoste, S. Béchu, J. Sirou, S. Miraglia, D. Fruchart, Plasma Phys. and Contr. Fusion., 47, A153 (2005)

4. A. Anders, Cathodic Arcs: From Fractal Spots to Energetic Condensation (Springer, New York, 2008)

5. A. Anders, J. Appl. Phys., 121, 171101 (2017)

6. J.C. Avelar-Batista, E. Spain, J. Housden, A. Matthews, Surf. Coat. Technol., 200, 1954 (2005)

7. S. Grigoriev, A. Metel, NATO Science Series, Series II: Mathematics, Physics And Chemistry, 155, 147 (2004)

8. C. Ruset, E. Grigore, Surf. Coat. Technol., 156, 159 (2002)

9. C. Ruset, E. Grigore, G.A. Collins, K.T. Short, F. Rossi, N. Gibson, H. Dong, T. Bell, Surf. Coat. Technol., 174-175, 698 (2003)

10. E. Grigore, C. Ruset, K. Short, D. Hoeft, H. Dong, X.Y. Li, T. Bell, Surf. Coat. Technol., 200, 744 (2005)
11. A.S. Metel, V.P. Bolbukov, M.A. Volosova, S.N. Grigoriev, Yu.A. Melnik, Instrum. Exp. Tech., 57, 345 (2014)

12. M. Volosova, S. Grigoriev, A. Metel, A. Shein, Coatings, 8, 287 (2018)

13. A.V. Hayes, V. Kanarov, B. Vidinsky, Rev. Sci. Instrum. 67, 1638 (1996)

14. E.M. Oks, A.V. Vizir, G.Yu. Yushkov, Rev. Sci. Instrum. 69, 853 (1998)

15. A.S. Metel, S.N. Grigoriev, Yu.A. Melnik, V.P. Bolbukov, Instrum. Exp. Tech., 55, 288 (2012)

16. S.N. Grigoriev, Yu.A. Melnik, A.S. Metel, V.V. Panin, Instrum. Exp. Tech., 52, 602 (2009)

17. A. Metel, S. Grigoriev, Yu. Melnik, V. Panin, V. Prudnikov, Japan. J. Appl. Phys., 50, 08JG04 (2011)

18. A.S. Metel, S.N. Grigoriev, Yu.A. Melnik, V.V. Panin, Plasma Phys. Rep., 35, 1058 (2009)

19. M.A. Lieberman, A.J. Lichtenberg, Principles of Plasma Discharges and Materials Processing (Hoboken: John Wiley \& Sons, 2005)

20. I. Langmuir, Phys. Rev., 33, 6 (1929)

21. E.W. McDaniel, Collision Phenomena in Ionized Gases (Willey, New York, 1964)

22. A.V. Phelps, J. Phys. Chem. Ref. Data., 20, 557 (1991)

23. A.V. Phelps, C.H. Greene, J.P. Burke, J. Phys. B: At. Mol. Opt. Phys., 33, 2965 (2000)

24. M. Kaminsky, Atomic and ionic impact phenomena on metal surfaces (Springer-Verlag, Berlin, 1965)

25. I. Danilov, M. Hackert-Oschätzchen, M. Zinecker,G. Meichsner, J. Edelmann, A. Schubert, Micromachines, 10, 214 (2019)

26. A.M. Chirkov, A.P. Rybalko, Ju.I. Rogalskij, et al, Patent of the Russian Federation No. 2381094 of 10.02.2010

27. N.N. Koval, A.D. Teresov, Y.F. Ivanov, E.A. Petrikova, Patent of the Russian Federation No. 2619543 of 16.05 .2017

28. V.P. Kudrya, Yu.P. Maishev. Mikroelektronika, 47, 51 (2018) 